\title{
Implementation and Evaluation of A New Model of Online, Facilitated, Peer Group Supervision for Dietitians Working in Eating Disorders: A Descriptive Overview Informed by The Kirkpatrick Training Evaluation Framework
}

Amanda Davis ( $\square$ amanda.davis@health.qld.gov.au )

Queensland Eating Disorder Service, Metro North Hospital and Health Service.

Nina Meloncelli

Royal Brisbane and Women's Hospital

Amy Hannigan

Queensland Eating Disorder Service, Metro North Hospital and Health Service.

Warren Ward

Queensland Eating Disorder Service, Metro North Hospital and Health Service.

\section{Research Article}

Keywords: online peer group supervision, dietitian, eating disorders, credentialing, practice standards, clinical supervision, ANZAED

Posted Date: February 7th, 2022

DOI: https://doi.org/10.21203/rs.3.rs-1319215/v1

License: (c) (1) This work is licensed under a Creative Commons Attribution 4.0 International License. Read Full License 


\section{Abstract}

\section{Background}

The recently published Australia and New Zealand Academy of Eating Disorders (ANZAED) practice and training standards for dietitians providing eating disorder treatment identified gaps in training for dietitians and recommended dietitians working in eating disorders (ED) seek further clinical experience, training, and supervision to provide effective, evidence- informed treatment. However, access to dietetic clinical supervision is problematic, secondary to limited trained supervisors, location, cost, and lack of organisational support. Demand for clinical supervision to expected to increase with the introduction of credentialing for ED clinicians in 2022. In 2018, QuEDS piloted an innovative model of group, online, facilitated and structured peer supervision with the goal of improving access to ED-specific clinical support. Positive evaluation of the QuEDS Facilitated Peer Group Support (QuEDS F-PGS) pilot led to a broader rollout of an additional four groups which were evaluated to formalise the F-PGS model and demonstrate its utility, acceptability, and implementation.

\section{Methods}

Groups consisted of 10 Queensland-based dietitians from public hospital, community or private practice with a Facilitator and Co-facilitator. Monthly 90 minute sessions were moderated, structured, and consisted of complex case discussions plus focus topic presentations. Participant experience was evaluated with anonymous, voluntary surveys at baseline, 6 months, plus a one-off survey in August 2021 informed by the Clinical Supervision Evaluation Questionnaire and Kirkpatrick's 4 level model of training evaluation. Program implementation was evaluated using the RE-AIM framework.

\section{Results}

Participant survey responses were highly positive across the four Kirkpatrick training evaluation domains of reaction, learning, behaviour, and results. Model implementation was positively evaluated with respect to reach, efficacy, and implementation. Respondents indicated they would recommend F-PGS to other dietitians and $96 \%$ planned to continue with the programme, a positive indicator of program sustainability.

\section{Conclusions}

QuEDS F-PGS program effectively utilised limited resources to provide more than 50 dietitians located in rural/regional and metropolitan areas with monthly, equitable access to ED-specific expertise and clinical support. QuEDS F-PGS is a cost-effective framework of peer group support which positively impacts dietitian's confidence and ability to deliver dietetic interventions in the ED arena and by inference the dietetic care of people with eating disorders.

\section{Background}


Provision of eating disorder (ED) treatment in Australia is problematic secondary to lack of access to specialist ED services, which are traditionally based in metropolitan hubs. Clinicians in generalist services (often lacking ED-specific training) provide first point of care, especially in rural/regional areas [1]. There has been a recent upsurge of interest in clinical supervision for allied health professionals since the release of the Australia New Zealand Academy of Eating Disorders (ANZAED) eating disorder treatment principles and general clinical practice and training standards and planned implementation of a credentialing system for clinicians working in eating disorders. Credentialing standards include specified levels of training, ongoing professional development and planned clinical supervision [2]. Professional development is key to maintaining and enhancing the knowledge and skills needed to deliver professional standards of service [3]. Mentorship and clinical supervision have been identified as important tools for professional development. However, there is a dearth of studies available on the effectiveness of group clinical supervision for allied health [4].

Inadequate undergraduate training of health professionals (including dietitians) in eating disorders has been identified as a workforce challenge with impacts on patient care [3]. Lack of ED-specific clinical confidence [5] may 'create stigmatised attitudes towards patients with EDs and impede the clinical care process' by delaying or obstructing appropriate treatment referrals [6]. Lack of clinical experience, 'especially in the area of ED management principles, may cause unintentional harm or even delay recovery' [3].

The recently published 'ANZAED Practice and Training Standards for Dietitians providing eating disorder treatment'identified gaps in training for dietitians working in this field, including communication/nutrition counselling skill deficits, and recommended dietitians working in EDs seek further clinical experience, training, and professional supervision to best provide effective, evidence-informed treatment [7]. Clinical supervision may be implicated in positive ED treatment outcomes [8] with a role in transforming novice practitioners into ED-specialists [9]. A recent systematic review suggested positive organisational outcomes including lower burnout and greater staff retention [10].

The Queensland Eating Disorder Service (QuEDS) is a statewide eating disorder service that provides training, consultation, and advice to clinicians (in addition to some evidence-based treatment services for people with eating disorders). The multi-disciplinary team at QuEDS assists health professionals and treating teams (in both public and private arenas) to upskill in the treatment of people affected by eating disorders. This is achieved by QuEDS's development and dissemination of treatment guidelines, education to health professionals, team to team case consultations and ad hoc support, supervision and mentoring of health professionals and treating teams. Dietitian-specific support has been provided via individual supervision (as per the predominant model of clinical supervision in allied health [11]), ad hoc peer to peer mentoring, targeted education, and creation of dietetic peer networks such as the Collaboration of Dietitians in Eating Disorders (COD-ED). However, these strategies are reliant on QuEDS's limited resources and expertise and the growing demand for supervision became unsustainable. 
Historically, clinical supervision has not been highly utilised in the field of dietetics [12]. Anecdotally, the QuEDS consultation service understood dietitians were seeking ED-specific support and training, similarly reported in a recent review [9]. A 2017 QuEDS survey (HREC/17/QRBW/202) of dietitians from nine Queensland hospitals found that although respondents felt 'well-supported' by QuEDS, the majority requested further educational opportunities, especially more accessible options such as on-line training and webinars. (report Quality Activity - Queensland Eating Disorder Service QuEDS Consultation Service. Improving quality of QuEDS-CS to public hospital dietitians. Available on request from author).

In 2018, in response to requests to QuEDS for clinical dietetic support and aligned with recommendations from National Agenda for Eating Disorders Practice standards, that clinicians should have access to EDspecific supervision, QuEDS conducted and evaluated a 12-month pilot of an innovative model of online group supervision [13]. The structure of the pilot model of QuEDS Facilitated Peer Group Support (QuEDS F-PGS) was informed by the knowledge that online training is effective and improves accessibility especially for rural/regional participants [14], and formal arrangements/structure enhance the processes and outcomes of peer group supervision [15]. The QuEDS F-PGS model of supervision was developed to be congruent with the definition of supervision as a 'formal activity for professional development and learning where there is an emphasis on discussion, feedback, guidance and support with the aim of enhancing the functionality, quality and capability or effectiveness of the supervisee' [16]. In addition, principles of adult learning theory (ALT) (self-directed, acknowledged experience and knowledge, goaloriented, relevant, practical, respectful) were utilised in development of the F-PGS format [17].

QuEDS F-PGS differed from usual model of peer group supervision (PGS) for allied health professionals which aligned with the New Zealand Coaching and Mentoring Centre model [18]. It was entirely online to facilitate access for dietitians outside of metropolitan areas and encouraged mixed groups with respect to experience levels and locations/departments to promote more open sharing of experiences. It was highly structured (and scripted) to ensure all participants equal opportunity to participate and be supported, and was assertively facilitated to ensure safe, evidence-informed practice was propagated (as dietetic intervention in EDs is considered to require high-level clinical knowledge), fidelity to the model (especially avoidance of didactic advice-giving), adherence to time allocations, and identification of insession learning opportunities. The role of QuEDS F-PGS Facilitator is stable and unlike PGS does not rotate amongst participants. The aim of the QuEDS F-PGS programme was to deliver acceptable, accessible, low-cost peer group support to Queensland-based Dietitians providing dietetic interventions to patients with eating disorders and increase their clinical knowledge and confidence. The QuEDS F-PGS pilot program provided a closed group of 10 dietitians (with one Facilitator and a Co-facilitator) monthly peer group supervision of 90 minutes including a 30 minute focus presentation, at nil cost. Participants were encouraged to complete de-identified surveys for evaluation of the programme and to prioritise session attendance. The overwhelmingly positive evaluation of the 2018/2019 pilot group informed the roll out of an additional four QuEDS F-PGS groups for dietitians working with eating disorder clients. The goal of the broader roll out of the QuEDS F-PGS program was to utilise QuEDS 's limited resources to provide clinical support to as many dietitians working in ED as possible, in alignment with QuEDS's mission to facilitate safe, effective treatment for people with ED throughout Queensland. 
Here, we provide a description and evaluation of the broader implementation of the QuEDS F-PGS model,12 months after the pilot program. Emphasis is on F-PGS utility, acceptability, and sustainability as a cost-effective model of provision of clinical support to clinicians working in the field of eating disorders. Detailed description of the process is provided to encourage replicability and comparison with other models.

\section{Methods}

\section{The Facilitated Peer Group Support Model}

Following the success of the pilot QuEDS F-PGS program (May 2018 to April 2019), an additional four FPGS groups were launched and evaluated for the purposes of this project. QuEDS F-PGS model consisted of online group sessions, each of 10 participants, plus a Facilitator and Co-Facilitator. Participation in the groups was invited through expression of interest documents (EOI) which were broadly distributed throughout Queensland. EOls were emailed to dietetic leadership groups (Dietitian Nutritionist Strategic Coalition Queensland and Queensland Health Nutrition Dietitian Leadership Coalition), and dietetic networks including Collaboration of Dietitians in Eating Disorders (COD-ED) and QuEDS's contact lists including QuEDS F-PGS waitlists and the QuEDS Newsletter listing. EOls included information on EDspecific clinical practice areas of groups, timings, and summary of session format. Applicants nominated the group they wished to join. Applicants signed an agreement detailing the importance of confidentiality and attendance which required countersignature from direct line management, to ensure support for attendance.

The model consisted of 90 minute sessions run monthly via calendar invites at regular times established in the initial EOI. Sessions were highly structured, facilitated and documented by the facilitators [see Additional file 1 session format with script.pdf]. Details of the groups, including clinical practice areas and launch dates are described in Table 1: Clinical Practice Areas and Launch Dates.

\section{QuEDS F-PGS Facilitation}

The QuEDS F-PGS Lead Facilitator, who developed the F-PGS model, was responsible for overarching administration of the PGS groups including Facilitator training, mentoring and support, EOI distribution, survey link distribution, documentation bundles and evaluation. Facilitators were recruited and provided with orientation by the Lead Facilitator and were expected to have more than 5 years clinical experience within the clinical focus area and have ideally attended clinical supervision training and participated in QuEDS F-PGS for at least 6 months. Essential skills included: understanding of the application of professional boundaries and risk management for the clinical focus area [see Additional file 2 facilitators guide.pdf]. The role of the Facilitator/Co-facilitator was moderation of the F-PGS sessions to ensure accurate documentation, time keeping and fidelity to the QuEDS F-PGS model. The Facilitator actively fostered safe practice, group cohesion, an atmosphere of support, acceptance and collegiate respect and increase in participants' confidence. They ensured appropriate sharing of experience without descent into didactic advice provision (including by the Facilitator), identified learning opportunities within session, 
and fostered reflective practice. Facilitators avoided the role of 'expert' within the group, whilst ensuring best practice was propagated through participants sharing experience and if necessary guided discussions to achieve this.

\section{QuEDS F-PGS Participants}

QuEDS F-PGS model consisted of one Lead Facilitator and 5 additional Facilitator/Co-Facilitators, each attached to specific clinical practice groups, providing 5 ongoing groups each month with total of $>50$ participants.

Participants were invited to apply if they were a Queensland-based Accredited Practicing Dietitian, in either the public health or private domain, with an interest in treating, or currently treating people with eating disorders, and the ability to commit to attendance of at least 10 out of 12 sessions annually. Applications were accepted on a first-come, first-served basis. Unsuccessful applicants were added to the QuEDS F-PGS waitlist and emailed EOI documents as vacancies arose secondary to withdrawals.

\section{Adaptations through implementation process}

Changes to the model during the broader implementation of QuEDS F-PGS were informed by ongoing insession feedback, online surveys, reflective practice of facilitator role, and to ensure model fidelity. These adaptations included:

- Change from Telehealth to Microsoft Teams for ease of delivery

- A shift from a 12 month duration to ongoing, secondary to participants wish to continue

- Increase in structure/scripting in response to feedback and to ensure fidelity to the model with roll out to additional groups/facilitators

- PGS Tools (NZ Coaching \& Mentoring Centre publication available www.coachingmentoring.co.nz) including 'veridical reports', 'good news analysis' etc., were discarded as participants chose not to use the tools

- Focus Topic - evolved from journal reviews/large topic discussion to didactic presentations prerecorded to align across all 5 groups

\section{Evaluation}

Participants (excluding those who had taken part in the pilot program) were invited to complete surveys at pre-commencement (baseline) [see Additional file 3 baseline survey.pdf] and follow-up at 6 months [see Additional file 4 follow up survey.pdf]. Survey links for pre-commencement (baseline) and 6-month (follow-up) surveys were emailed to current participants at time points as triggered by documentation of start dates. Surveys were anonymous and voluntary. Linkage between surveys was attempted through generation of a personalised code. Participants who withdrew from F-PGS prior to 6 months participation were not invited to complete the follow-up survey. Surveys were designed to qualitatively evaluate the acceptability and utility of the model. In recognition of the need to identify the impact of F-PGS on 
changes to clinical practice, a decision was made to conduct a 'one off' Learning and Clinical Practice Survey (LCP Survey) in August 2021 [see Additional file 5 LCP survey.pdf].

All participants (including withdrawals) from the initiation of the program in 2018 until August 2021 were invited to respond to the LCP Survey.

For purposes of evaluation, the baseline, follow-up, and LCP Surveys were compared.

Baseline and follow-up surveys were developed with reference to the Clinical Supervision Evaluation Questionnaire (CSEQ) [19], the Metro North Hospital and Health service Peer Supervision Group Evaluation form (informed by NZ Coaching and Mentoring Centre), previous surveys from the QuEDS FPGS pilot program and the Kirkpatrick Model for Training evaluation (REF). An overview of survey development is available [see Additional file 6 survey development.pdf], which includes relevant Kirkpatrick levels of training evaluation [20]. The LCP survey was informed by the four-area Kirkpatrick training evaluation model and designed to capture additional data on self-assessed change in clinical practice directly attributed to participation in F-PGS. Implementation of the program was evaluated using the RE-AIM framework [19].

\section{Data Analyses}

Results from each survey type (baseline, follow-up, and LCP surveys) were reported descriptively under the relevant Kirkpatrick Level where applicable (See Table 3). Not all baseline and follow-up surveys were able to be linked (for example: inconsistent personalized codes, different respondents to each survey). Therefore, the pooled results were reported for each survey, and no attempt was made to infer within person changes to any of the Kirkpatrick Levels. However, to understand the change in confidence between baseline and follow-up surveys for linked responses that were able to be determined, a regression model was used to examine the association between confidence and survey time (baseline and follow-up). A mixed model with a random intercept per person was utilised to account for the expected within-person correlation. Not all respondents provided two responses. The adequacy of the normal assumption was tested by plotting the model residuals, which provided strong evidence that there was no need to use non-parametric statistics.

Qualitative free text responses from all surveys were grouped according to common themes. Example quotes are used to highlight participant's responses under each Kirkpatrick Level.

\section{Results}

F-PGS A (original pilot group) transitioned as a 'business as usual' group from May 2019. F-PGS B and C commenced in September 2019 and F-PGS D and E commenced in January 2021.

There were 76 total new recruits to the program following the pilot group (May 2019 to August 2021). All groups have functioned at $100 \%$ capacity (10 participants +2 facilitator/co-facilitators) since inception, excepting F-PGS E (commenced with 6 participants +2 Facilitators/Co-facilitators) due to relative lower 
numbers of dietitians working in community mental health positions. Recruitment to vacancies is reflected in the higher total numbers below. Total group numbers are shown in Table 1 below.

Table 1: Clinical Practice Areas and Launch Dates

\begin{tabular}{|llll|}
\hline GROUP & COMMENCE & $\begin{array}{l}\text { CLINICAL FOCUS AREA WITHIN EATING } \\
\text { DISORDERS }\end{array}$ & TOTAL PARTICIPANTS \\
\hline $\begin{array}{l}\text { F-PGS } \\
\text { A }\end{array}$ & May 2019 & QId health dietitians - general ED focus & $\begin{array}{l}19 \text { (includes 10 pilot } \\
\text { participants) }\end{array}$ \\
\hline $\begin{array}{l}\text { F-PGS } \\
\text { B }\end{array}$ & $\begin{array}{l}\text { September } \\
2019\end{array}$ & Private practice dietitians ED focus & 23 \\
\hline $\begin{array}{l}\text { F-PGS } \\
\text { C }\end{array}$ & $\begin{array}{l}\text { September } \\
2019\end{array}$ & Paediatric/adolescent dietitians ED & 20 \\
\hline $\begin{array}{l}\text { F-PGS } \\
\text { D }\end{array}$ & $\begin{array}{l}\text { January } \\
2021\end{array}$ & Private practice dietitians ED focus & 13 \\
\hline $\begin{array}{l}\text { F-PGS } \\
\text { E }\end{array}$ & $\begin{array}{l}\text { January } \\
2021\end{array}$ & Community ED focus & 11 \\
\hline
\end{tabular}

There were 27 withdrawals from the F-PGS program during the evaluation period between May 2019 and August 2021. Ten participants withdrew prior to six months' participation and did not complete the follow-up survey. Reasons for withdrawal were documented. Nine participants withdrew secondary to parental leave and 18 due to workload/position change.

The baseline survey was sent to all participants and completed by 59 F-PGS participants $(77.6 \%$ response rate). The follow-up survey was completed by 37 participants ( $56 \%$ response rate). The LCP Survey was sent to all past and current QuEDS F-PGS participants (including pilot group) with valid email addresses (70) and received 50 responses (71\% response rate). Participant characteristics are shown in Table 2 (Characteristics of survey respondents) for all three surveys. Of the follow-up survey respondents, 28 (76\%) could be matched to the baseline survey. The LCP survey was not intended to be matched to previous surveys. A greater proportion of the total responses to the baseline and follow up survey were from group $B$ and $C$ participants, reflecting the larger number of participants through these groups (longer duration than groups $D$ and $E$ ) and the exclusion of pilot participants (original participants of F-PGS A) from the baseline and follow-up surveys. Almost $3 / 4$ of respondents ( $73 \%$ baseline, $76 \%$ follow-up) were from a Metro centre or capital city (table). More than $20 \%$ of participants were from rural and remote centres. Around half the respondents in both groups ( $46 \%$ and $54 \%$ ) reported having less than 5 years clinical experience, with more than $20 \%$ greater than 10 years' experience.

Table 2. Characteristics of survey respondents 


\begin{tabular}{|c|c|c|c|}
\hline & $\begin{array}{l}\text { Baseline } \\
\text { Survey }\end{array}$ & $\begin{array}{l}\text { Follow-up } \\
\text { Survey } \\
\text { (6months) }\end{array}$ & $\begin{array}{l}\text { Learning and Clinical practice } \\
\text { survey }\end{array}$ \\
\hline Respondents & $\begin{array}{l}59 \\
(78 \%)\end{array}$ & $37(56 \%)$ & $50(71 \%)$ \\
\hline \multicolumn{4}{|l|}{ F-PGS } \\
\hline A (general focus) & $7(12 \%)$ & $3(8 \%)$ & $11(22 \%)$ \\
\hline B (private practice) & $\begin{array}{l}16 \\
(27 \%)\end{array}$ & $9(24 \%)$ & $13(26 \%)$ \\
\hline C (adolescent focus) & $\begin{array}{l}16 \\
(27 \%)\end{array}$ & $8(22 \%)$ & $8(16 \%)$ \\
\hline D (private practice) & $\begin{array}{l}11 \\
(19 \%)\end{array}$ & $6(16 \%)$ & $10(20 \%)$ \\
\hline E (community focus) & $6(10 \%)$ & $4(11 \%)$ & $8(16 \%)$ \\
\hline Not stated & $3(5 \%)$ & $7(19 \%)$ & \\
\hline \multicolumn{4}{|l|}{ Location } \\
\hline Metro & $\begin{array}{l}22 \\
(37 \%)\end{array}$ & $13(35 \%)$ & \\
\hline Capital city & $\begin{array}{l}21 \\
(36 \%)\end{array}$ & $15(41 \%)$ & \\
\hline Rural & $\begin{array}{l}12 \\
(20 \%)\end{array}$ & $7(19 \%)$ & \\
\hline Remote & $3(5.1 \%)$ & $2(5.4 \%)$ & \\
\hline Not stated & $1(1.7 \%)$ & & \\
\hline \multicolumn{4}{|l|}{ Experience } \\
\hline$<5$ years & $\begin{array}{l}27 \\
(46 \%)\end{array}$ & $20(54 \%)$ & \\
\hline $5-10$ years & $\begin{array}{l}19 \\
(32 \%)\end{array}$ & $8(22 \%)$ & \\
\hline$>10$ years & $\begin{array}{l}13 \\
(22 \%)\end{array}$ & $9(24 \%)$ & \\
\hline \multicolumn{4}{|c|}{ Number of clients (past 12 months) } \\
\hline$<5$ clients & $\begin{array}{l}21 \\
(36 \%)\end{array}$ & $10(27 \%)$ & \\
\hline 5 - 15 clients & $\begin{array}{l}21 \\
(36 \%)\end{array}$ & $13(35 \%)$ & \\
\hline
\end{tabular}


$>15$ clients

\section{Place of employment}

Public Hospital

$24 \quad 17(46 \%)$

$(41 \%)$

Public Community Health Centre

$8(14 \%) \quad 9(24 \%)$

Private Hospital

$8(14 \%) \quad 3(8.1 \%)$

Private Practice

30

(51\%)

$18(49 \%)$

2 (3\%) $\quad 1(2.7 \%)$

Service

University Clinic

$1(2.7 \%) \quad 0(0 \%)$

Public Specialist Eating Disorder

Non-government organization

$4(7 \%) \quad 1(2.7 \%)$

Dietetic student

$1(2.7 \%)$

\section{Client Group}

Paediatric

20

(34\%)

Adolescent

46

$(78 \%)$

Adult

$8(22 \%)$

$27(73 \%)$

$35(95 \%)$

\section{Kirkpatrick Level 1: Reaction}

Overwhelmingly, participants reported positive experiences with QuEDS F-PGS sessions across all Reaction domains (Table 3). Participants reported high levels of feeling 'safe', maintenance of 'confidentiality', positive feelings of 'confidence', feeling 'supported' and positive impact of facilitation.

Table 3. Responses according to the four levels for Kirkpatrick's Model of Evaluation. 


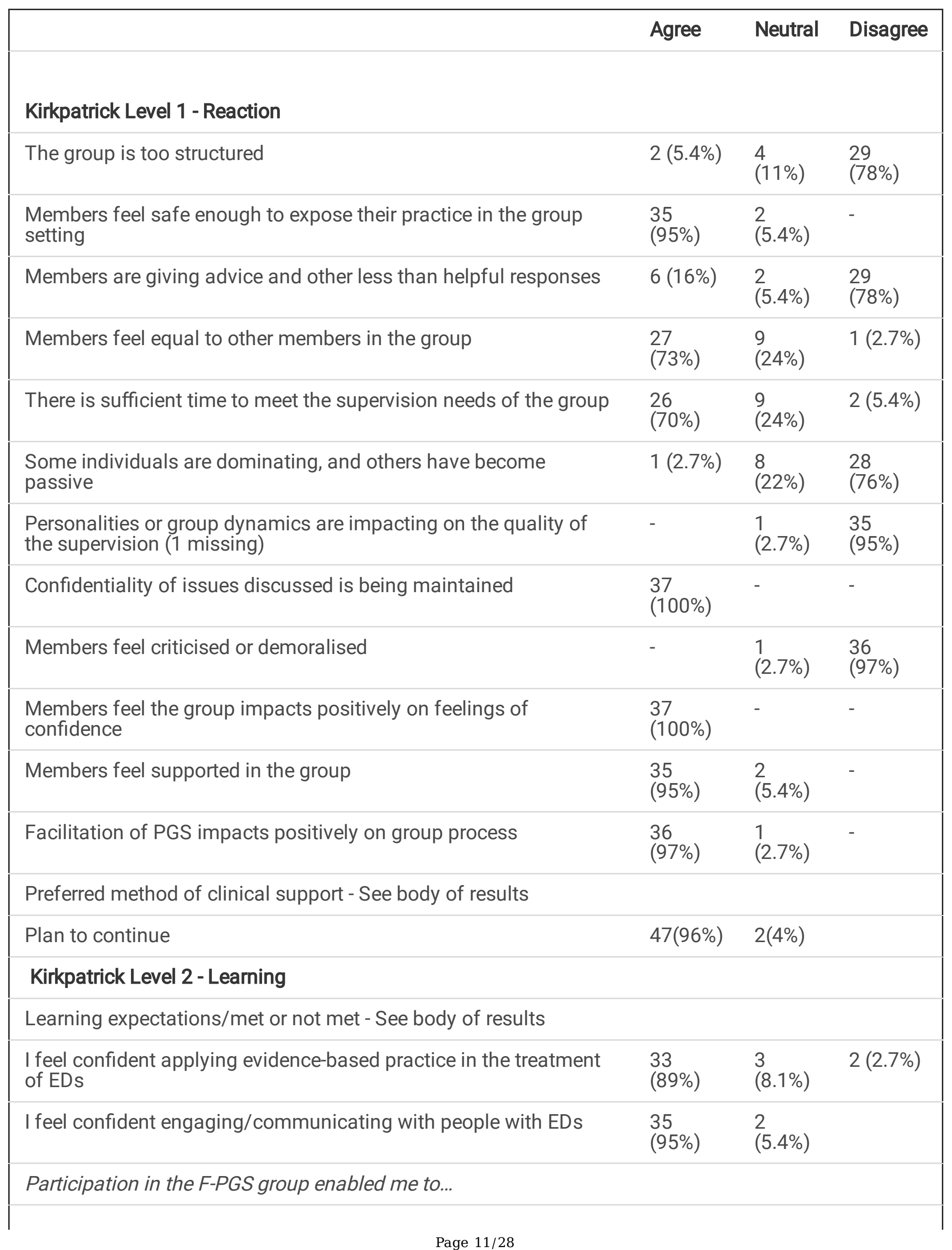


To increase my clinical knowledge/skills

To increase my supervisory/mentoring skills

To meet ongoing professional development requirements
49

$(98 \%)$

\section{3}

$(67 \%)$

42

$(84 \%)$
$1(2 \%)$

14

$(28 \%)$

$3(6 \%)$

8

$(16 \%)$

\section{Kirkpatrick Level 3 - Behaviour/Implementation of Learnings}

F-PGS has changed my clinical practice as evidenced by...

More appropriate implementation of evidence-based

practice/guidelines

44

$(88 \%)$

6

Application of ED-specific resources/tools

45

$(90 \%)$

4

$(11 \%)$

Increased ability to provide dietetic intervention for complex ED cases

Participation in the F-PGS group enabled me to...

To increase my reflective practice

44

$(88 \%)$

$\stackrel{6}{(12 \%)}$

\section{Kirkpatrick Level 4 - Results/Broader Impacts}

I feel supported as a dietitian working in the field of EDs

31

$(84 \%)$
4

$(11 \%)$

$2(5.4 \%)$

Participation in the F-PGS group enabled me to...

To become more confident in my clinical work

To feel supported in my clinical work 48

$(96 \%)$

49

$(98 \%)$

To cope better with stressors of working with ED clients

To better enjoy my work in the ED arena

43

$(86 \%)$

$2(4 \%)$

42

$(84 \%)$

$1(2 \%)$

47

$(94 \%)$

To achieve more in my ED-specific clinical work

44

$(88 \%)$

Increased advocacy for appropriate care for ED clients

\section{5}

$(90 \%)$

Active engagement in ED-specific service development

40

$(80 \%)$
6

$(12 \%)$

(10\%)

5

$(10 \%)$

7

$(14 \%)$

$2(4 \%) \quad 1(2 \%)$

(18\%) 
Participation in QuEDS F-PGS has led me to change my clinical practice

\section{Sustainability}

I would like to continue with QuEDS F-PGS

I would recommend QuEDS F-PGS to other dietitians

I prefer the format of QuEDS F-PGS to standard Peer Group Supervision

QuEDS F-PGS is a valuable adjunct to my clinical supervision/mentoring

The QuEDS F-PGS model would be appropriate for other clinical areas/disciplines
49

$(98 \%)$

$1(2 \%) \quad-$

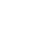

48

$2(4 \%) \quad-$

50

(100\%)

\section{6}

49

48

(96\%)

$4(8 \%) \quad-$

$1(2 \%) \quad-$

$2(4 \%) \quad-$

Some participants provided suggestions for improvement:

- 'To facilitate more time for case discussion, it may be helpful for members to submit a short SBAR and their related support question to the facilitator 1 week prior. The members can then be sent the list of available case reviews and indicate their preference. This may help save time at the start of the session.'

- Dietitian participant group unknown

'Individual supervision is also beneficial for different reasons. I wonder if the program could be expanded to offer (less frequent) individual support also?'

- Adolescent eating disorder dietitian participant in F-PGS C

'I feel quite daunted in the group setting sharing my experiences or feeling I don't have the level of experience of the others in my group.'

- Community dietitian participant in F-PGS E

Examples of positive reactions to the F-PGS as reported by the participants include:

'I found it intimidating giving comments at times... when making comments l'd freeze or jumble my words lol. With time I gained more confidence when speaking so that was wonderful to experience and slowly overcome thank you!!'

- Public hospital dietitian participant F-PGS A 
If found the QUEDS FPGS group to be beneficial in many ways. The facilitator has provided a good amount of structure and focus topics that are relevant to practice, including a broad spectrum of disorders and presentations. The opportunity to connect with peers working in the same field has reduced my feels of isolation and also allowed my confidence to grow as I learn that many of my peers experience similar fears and insecurities.'

- Private dietitian participant in F-PGS

'I think it is a fantastic model that provides access to a quality supervision model that can be accessed at no cost to the member. Love the idea of learning from peers and feel the model has been a wonderful addition to the ED landscape for dietitians.'

- Dietitian participant group unknown

'I attend multiple supervisions regularly with the others being expert-led rather than peer group and I find this model to be the most supportive.'

- Dietitian participant in adolescent group F-PGS C

'I have really enjoyed the format - case discussion followed by structure PD at the end.'

- Dietitian participant in adolescent group F-PGS C

'The changes made over the last 12months where the number of groups have been expanded to focus on more individualized practice areas has meant the sessions are increasingly streamlined and allow for more meaningful conversations as well as the inclusion of a focus topic presentation.'

- Community-based dietitian participant F-PGS E

Participant's reactions to the QuEDS F-PGS model (in the follow-up survey) with respect to preferred mode of upskilling in dietetic intervention for ED were ranked from most to least preferred as follows: Individual supervision, QuEDS F-PGS, specialist education sessions, workshops, guidelines, peer group supervision, online education modules. Ninety-two percent of respondents in the LCP survey preferred the QuEDS FPGS format to standard PGS (peer group supervision).

\section{Kirkpatrick Level 2: Learning - self-assessed increase in skills}

Learning was assessed in the follow-up survey and the LCP survey. Confidence ratings and reported increased skills are shown in Table 3 (Responses according to the four levels for Kirkpatrick's Model of Evaluation), while learning expectations are shown in Table 4. For most participants, learning expectations were met across all domains. From the LCP survey [see Additional File 4], of the 50 respondents, the number of respondents reporting unmet learning expectations (from highest to lowest) are as follows: ED-specific counselling skills $(n=6)$; ED-specific evidence-based practice/guidelines $(n=4)$; assessment/treatment of ED diagnoses ( $n=4)$; ED-specific tools/resources ( $n=3)$; Complex ED case 
management $(n=2)$; ED presentations ( $n=1)$; confidence to implement ED-specific dietetic interventions $(n=1)$. Overall, the free text responses were very positive with minimal suggestions for improvement to enhance learning:

'I would like to note that I felt these have not been met yet as I believe these are ongoing skills and I do not think they will ever feel 'met'. Additionally, there is a lot to cover and so the groups should not be the only place where we are seeking this information and learning.'

- Community dietitian participant in F-PGS E

'I also wanted to learn how other dietitians approached situations and learn from them in the group.'

- Private dietitian participant in F-PGS B

'I have learnt new skills and had exposure to a wide variety of focus topics and PD opportunities.'

Private dietitian Participant F-PGS D

Table 4. Learning expectations as reported by eating disorder dietitians in the learning and clinical practice (LCP) survey $(\mathrm{n}=50)$

\begin{tabular}{|lcc|}
\hline & $N$ & $\%$ \\
\hline Knowledge of ED-specific evidence-based practice/guidelines & 28 & $56 \%$ \\
\hline Knowledge of ED-specific resources/tools & 43 & $86 \%$ \\
\hline Clinical knowledge of eating disorder presentations & 29 & $58 \%$ \\
\hline Knowledge of assessment/treatment of ED diagnoses & 30 & $60 \%$ \\
\hline ED-specific counselling skills & 35 & $70 \%$ \\
\hline Understanding of formulation of management plans for complex ED clients & 28 & $56 \%$ \\
\hline Confidence in ED-specific dietetic interventions & 41 & $83 \%$ \\
\hline Support from colleagues for your work in the ED arena & 41 & $83 \%$ \\
\hline
\end{tabular}

\section{Changes in confidence ratings}

Three questions in the follow-up survey could be assessed as pre-post changes to ratings. There was a similar increase in confidence for the adjusted and unadjusted models, therefore, only the results of the unadjusted regression modelling are provided. The mean change in confidence $(95 \% \mathrm{Cl})$ from pre- 
commencement (baseline survey) to post (6-month follow-up survey) was consistent across all three domains of communication, evidence-based practice and feeling of being supported in clinical work. For the statement 'I feel confident applying evidence-based practice in the treatment of eating disorders' $(E B P)$, the mean confidence rating was 0.7 (0.4 to 0.9$)$ higher in the follow-up survey. For the statement 'I feel confidence engaging/ communicating with people with eating disorders' (communication), the mean confidence was 0.6 (0.4 to 0.9) higher, and for the statement 'I feel supported as a dietitian working in the field of eating disorders', the mean confidence was 0.9 (0.6 to 1.2) higher in the follow-up survey (see Figure 1 below).

\section{Kirkpatrick Level 3: Behaviour - implementation of newly acquired skills/knowledge}

Most respondents (88\%) felt that they had changed their clinical practice by improved implementation of evidence-based guidelines and (90\%) application of ED-specific tools and resources (Table 3 ).

Participants (94\%) also felt they had increased their ability to provide dietetics intervention for complex ED cases, and 88\% agreed that participation in QuEDS F-PGS had increased their reflective practice (Table $3)$.

'QUEDS PGS has changed the way I provide care to eating disorder patients. Not only has QUEDS PGS contributed greatly to my knowledge and skills in ED-specific nutrition prescription, but also the monthly focus topic has broadened my understanding of medical complications, co-morbidities, MDT considerations, available resources, mental health and the patient journey. Listening to complex cases shared by the group provides incredibly valuable learning. Hearing the different perspectives and experiences of my colleagues has definitely broadened my thinking.'

- Public hospital dietitian participant in F-PGS A

\section{Kirkpatrick Level 4: Results - broader impacts of participation in F-PGS}

Participation in QuEDS F-PGS impacted on self-reported results across several domains. Respondents reported increased 'confidence' (96\%) in clinical work, improved 'engagement' (88\%) with, and increased 'advocacy for appropriate care', (90\%) for ED clients (Table 3).

Ninety-eight percent of respondents reported participation in QuEDS F-PGS enabled them to feel 'supported' in their clinical work, to cope better with 'stressors of working with ED clients' (86\%), and to better "enjoy work in the ED arena" (84\%). Ninety-four percent felt F-PGS had helped them to 'achieve more' and be more actively engaged in 'service development' in the ED arena.

Some of the respondents' comments that highlight this improvement in results include: 
'The QUEDS F-PGS has provided a very valuable source of support for gaining greater knowledge and confidence to be able to support consumers with eating disorders. As a Dietitian working in a regional hospital with a varied case load, I strongly value the opportunity to participate each month to gain support and learn from others working in the eating disorder space.'

- Public hospital dietitian participant in F-PGS A

'This group has helped my confidence in seeing eating disorder clients and increased my awareness of the supportive network of dietitians in the area. My skills in dietetic specific and in collaborative care have improved. This group has also been instrumental in my reflective practice and in maintaining my mental health whilst working in the area.'

- Community dietitian participant in F-PGS E

'Invaluable experience, unsure I would still be working in this field without the support.'

-Private dietitian participant in F-PGS D

'Excellent peer support which significantly improved my confidence and enjoyment of ED workload.'

-Private dietitian participant F-PGS B

'The biggest one is definitely the reassurance the team provides with difficult cases, to advocate and be confident in our evidence-based dietetic management.'

- Dietitian participant in adolescent F-PGS C

'The sessions have not only given me more confidence in treating EDs but have reignited my passion and interest in the area.'

- Private dietitian participant in F-PGS B

\section{QuEDS F-PGS implementation informed by RE-AIM}

Table 5. RE-AIM framework measures 


\begin{tabular}{|c|c|c|}
\hline $\begin{array}{l}\text { RE-AIM } \\
\text { framework }\end{array}$ & Measure & \\
\hline Reach & $\begin{array}{l}\text { Participant } \\
\text { characteristics }\end{array}$ & $\begin{array}{l}11 / 16 \text { possible Queensland Hospital Health Services } \\
\text { represented } \\
>25 \% \text { rural/remote clinicians } \\
\sim 50 \% \text { private practitioners }\end{array}$ \\
\hline Efficacy & $\begin{array}{l}\text { Impact on: } \\
\text { Clinician, } \\
\text { Patient, } \\
\text { Service outcomes }\end{array}$ & $\begin{array}{l}94 \% \text { clinicians increase ability to provide dietetic } \\
\text { interventions } \\
90 \% \text { increased advocacy for client care } \\
94 \% \text { increased involvement in ED-specific service } \\
\text { development activities }\end{array}$ \\
\hline Adoption & $\begin{array}{l}\text { Uptake by other } \\
\text { services, groups }\end{array}$ & Not yet demonstrated - interest from other services \\
\hline Implementation & $\begin{array}{l}\text { Fidelity to model } \\
\text { Cost to deliver }\end{array}$ & $\begin{array}{l}\text { Not measured } \\
\sim 22.5 \mathrm{hrs} \text { Lead Facilitator } \& \text { Facilitator/co-Facilitators } \\
\text { provides } \sim 75 \mathrm{hrs} \text { participant support }\end{array}$ \\
\hline Maintenance & $\begin{array}{l}\text { Participant: } \\
\text { Recruitment } \\
\text { Retention }\end{array}$ & $\begin{array}{l}86 \text { recruits to August } 2021 \\
27 \text { withdrawals } \\
9 \text { parental leave } \\
18 \text { workload/position change } \\
71 \% \text { respondents to voluntary LCP survey } \\
96 \% \text { intention to continue with F-PGS }\end{array}$ \\
\hline
\end{tabular}

Detailed documentation of participants and sessions in addition to the surveys, assisted in evaluating the implementation of the QuEDS F-PGS program using the RE-AIM framework. Table 5 provides a breakdown of the RE-AIM parameters. Reach of the program was demonstrated by drawing participants from 11 of the possible 16 Queensland Hospital and Health Service (HHS) areas, with more than $25 \%$ of participants were from rural/remote areas, and $\sim 50 \%$ of participants involved in private dietetic practice.

Efficacy or impact of the program on participants, as derived from the self-assessed surveys, suggested highly positive outcomes ( $>90 \%$ self-assessed) of increased ability to provide dietetic interventions, 
advocacy for client care and involvement in ED-specific service development activities. Direct patient and service outcomes were not measured. Uptake by other groups/services was not measured by this study. Implementation costs measured in clinician time were as follows: total Facilitator time (Lead Facilitator, group Facilitators and Co-facilitators) of $\sim 22.5 \mathrm{hrs}$ per month provided 5 groups of 10 participants with 90 min per month of clinical support. Program maintenance was demonstrated by participant retention as documented at time of withdrawal from the program. There were 27 withdrawals over the study period. The majority of withdrawals (18) were secondary to workload/position changes, with 9 participants taking parental leave. Strong participant engagement was determined by $96 \%$ intention to continue with F-PGS and high proportion of participants engaging in voluntary surveys $(71 \%)$.

I would love to see this model used for dietitians as it is accessible, supportive and I have learnt so much from being in a group with a varied level of experience and expertise. This model is definitely needed in this area of practice!'

Private dietitian participant in F-PGS B

'FPGS is the best thing to ever happen to me as a dietitian.'

Private dietitian participant in F-PGS B

'FAR more valuable than regular PGS. This is my favourite hour of the month. Incredibly useful, would strongly recommend to others!'

Public hospital dietitian participant in F-PGS A

'The support of the FPGS Groups has moved me from being a generalist dietitian who is interested at practicing from a Health at Every Size lens but was not even fully able to achieve this to a thriving ED service provider who has just been accepted into a role with an entire ED focus. I am also a fully committed advocate and provide in-service education to my colleagues. Without QuEDS I would not be where I $a m^{\prime}$

- Private dietitian participant F-PGS B

\section{Discussion}

The purpose of the current study was to describe the implementation and evaluation of the QuEDS Facilitated Peer Group Support (QuEDS F-PGS) model, which was developed to enable broader access to cost-effective, appropriate clinical supervision for dietitians working in eating disorders. The utility, acceptability and impact of the model were evaluated according to the four Kirkpatrick Levels for training evaluation. Participant responses to the evaluation surveys were uniformly positive across the 4 Kirkpatrick training evaluation domains of reaction, learning, behaviour, and results. 
This study showed QuEDS F-PGS outcomes aligned with goals of clinical supervision documented in the ANZAED clinical practice standards as evidenced by participants' self-assessed increase in ability to implement evidence-based dietetic care, increase in reflective practice, increased ability to advocate for best care for patients, and improved feelings of enjoyment and decrease in stress associated with the ED workload.

The ANZAED clinical practice standards state 'clinical supervision and ongoing professional development aim to upskill clinicians, support reflective practice, aid the provision of high-quality treatment, and recognise the intensity and personal impact of treating complex mental health issues' [2]. The familiarity of the PGS model (from NZ Coaching \& Mentoring Centre), which has been widely implemented and evaluated within Qld Health, provided the impetus to explore a modified format of group supervision to better suit the ED context and to meet the increasing demand for provision of highquality ED-specific dietetic supervision. Clinicians with skills honed by consultation liaison experience, are ideally placed to facilitate supervision groups [22]. QuEDS dietitians' consultation experience, including their knowledge of best clinical practice and risk management was an ideal skills-mix for facilitation of supervision groups, thereby increasing QuEDS's capacity to provide high quality, expert group supervision.

Demand for appropriate clinical supervision is expected to increase further with the introduction of credentialing for ED clinicians in 2022. Opportunity to increase the facilitator workforce through training provided by the Lead Facilitator and ongoing support of the facilitative process via the QuEDS F-PGS model structure (e.g., scripting), mentoring, and peer to peer facilitator support will continue to allow for program expansion into the future.

To the best of our knowledge, the QuEDS Facilitated Peer Group Support model is unique in the eating disorder management context and within the realms of supervision models for dietitians and allied health professionals. It inhabits the middle ground between two comparable models which have been delivered to allied health clinicians in the Australian or New Zealand health system context: Group Clinical Supervision (GCS) models with an identified skilled facilitator as locus of experience, and Peer Group Supervision (PGS) models which traditionally rotate facilitation roles through the participants. The QuEDS F-PGS Facilitator, whilst facilitating group process, model fidelity and ensuring only best practice advice is propagated, avoids stepping into the 'expert' role, thus aligning with principles of adult learning theory [17]. The QuEDS F-PGS model aimed to meet dual needs for clinical supervision and ongoing professional development through inclusion of a didactic focus topic in addition to peer discussion of cases/situations. Other models of group supervision do not specify inclusion of a focus topic presentation in the session format.

The PGS model, aligning with the NZ Coaching and Mentoring Centre framework, was evaluated by both Pager et al [23] and Kuipers et al [15] following a broad roll out of PGS training to Queensland Health staff (PGS-AH), and by Dungey et al [24] in a smaller training program delivered to 5 centres of radiation therapists in New Zealand (PGS-RT). In these studies clinicians were trained in the PGS model and 
encouraged to set up and/or participate in PGS groups in variously structured manners and their experience of such evaluated.

The conclusion that higher levels of structure increased group supervision effectiveness influenced Gardner et al [11] to trial a GCS model (specifically a critical reflection model) with non-rotating trained facilitators and a suite of documentation designed to increase structure including: an agenda template, set frequency and duration of sessions, and regular evaluation. Gardner's GCS model more closely approximates QuEDS F-PGS with respect to level of formal structure. QuEDS F-PGS documentation suite includes additional guidelines such as a facilitator guide document, orientation guide document, session format with script, session documentation format, participant attendance spreadsheets, centralized data storage, online surveys etc. Other F-PGS structures similarly include set frequency and duration of sessions and regular evaluations via surveys.

Ongoing themes in literature include the importance of structure and facilitative process on effectiveness of peer supervision, however, as highlighted in a recent systematic review of supervision studies, there appears to be a lack of adequate descriptions of the framework of supervision models in the literature diminishing both replicability of the models and understanding of their implementation and evaluation [10]. The detailed description of the QuEDS F-PGS program included in this evaluation addresses this issue.

The QuEDS F-PGS program was more structured than the aforementioned GCS and PGS models, including assured fidelity to the model secondary to the integral role of the Lead Facilitator supporting administration of the program and mentoring other facilitators, in addition to a formal documentation suite. Dietitians attending the QuEDS F-PGS responded positively to this formal, structured peer group support model in alignment with the PGS and GCS studies which have also demonstrated that structured processes and formal documentation enhance the processes and outcomes of clinical group supervision $[15,11]$.

PGS-AH groups with a higher level of structure and evaluation scored more highly for 'sense of safety', 'confidentiality', 'trust between members', with a more likely positive impact on the 'quality of their care', stress amelioration, and feelings of 'confidence' in their clinical work [15]. The structured nature of QuEDS F-PGS appears key to the high ratings respondents scored the QuEDS F-PGS process for 'safety', 'confidentiality', 'clinical confidence' and other group dynamics. It is presumed feelings of 'safety' and 'confidentiality' are indicative of group cohesion and contribute to longevity and success of supervision groups [25].

Conversely, lack of structure in other models negatively impacted group process and outcomes. Some PGS-RT groups for radiation therapists evaluated were negatively impacted by lack of leadership, group dynamics, fluctuating attendance etc., which did not engender a safe, supportive environment [24]. Issues in model delivery were reported by $\sim 30 \%$ of PGS-AH respondents including non-adherence to agenda, poor attendance, frequent membership changes and perception that professional support needs of members were not being met [23], which may negatively affect outcomes. 
In the survey of the GCS pilot to allied health participants in rural Victoria (3 groups of 4-8 participants in pilot with survey response rate of 58\%) respondents agreed that 'there were well-established ground rules in the group', and 'confidences I share will be respected'. However, participants also noted that the quality of the session 'was highly reliant upon the facilitator' [11]. An evaluation of reflective practice groups for mental health nurses identified the key role of the facilitator in ensuring a safe group environment [25]. The role of the QuEDS F-PGS Lead Facilitator promotes model fidelity and positively impacts acceptability and outcomes. QuEDS F-PGS had uniformly positive responses across groups with different facilitators. However, between group variation was not formally tested.

As with other supervision models [11,15], organisational support, which in the case of QuEDS F-PGS was provided by the utilitisation of the Queensland Health dietetic leadership to disseminate and promote the program, plus a requirement for line management approval to participate, was found to be essential for successful implementation of supervision.

In the Australian generalist medical model specialist services are usually located in metropolitan hubs. Rural generalist clinicians are required, of necessity, to deliver specialist care, often without adequate clinical support [14]. Accessibility to clinical supervision for rural clinicians, isolated both geographically and collegiately, is important to ensure appropriate clinical care. Collegiate isolation is also common within the private practice arena. Private practitioners (often inexperienced, without team support) are increasingly likely to encounter ED clients in the community [3] secondary to recent government incentives via Medicare Benefit Scheme Eating Disorder Management Plan. Our survey findings indicate the QuEDS F-PGS model provided support to rural/remote clinicians, who accounted for $~ 25 \%$ of participants, and was successfully implemented/accepted by private practitioners ( $50 \%$ participants) and enabled by online delivery at zero cost to participants.

Accessibility to online clinical support and supervision has become increasingly important since the COVID-19 pandemic but concerns as to its effectiveness must be addressed. QuEDS F-PGS commenced pre-COVID-19 and was deliberately delivered online to increase accessibility for rural/regional clinicians and to enable a broad mix of participation in each group. This study provided evidence that an entirely online model of peer supervision is acceptable and effective.

The QuEDS F-PGS model was deemed by $98 \%$ of participants to have impacted their clinical practice. The majority of survey respondents felt QuEDS F-PGS had helped them to 'achieve more' and be more actively engaged in 'service development' in the ED arena, and more effectively implement 'best practice'. In comparison, GCS surveys indicated less than average agreement that 'clinical supervision has definitely had a positive effect on the quality of care that I provide', although 2/3 (67\%) of GCS respondents still had a positive perception of group clinical supervision [11].

The QuEDS F-PGS differs from PGS-AH and GCS models (and their evaluation) in that it exclusively pertained to dietitians working in ED, as opposed to multiple disciplines and clinical areas of allied health and encompassed both public hospital and private practice dietitians. The PGS-RT evaluation demonstrated the effectiveness of PGS in single discipline groups [24]. QuEDS F-PGS evaluation 
demonstrated the applicability of a similar model across both public and private domains, but it has not been tested outside of the ED arena, or with multi-disciplinary groups or other single professional groups. It may be challenging to implement this model with multi-disciplinary groups whilst retaining the professional development (focus topic) facet of the model.

Health professionals working with ED patients may be particularly prone to burnout which may be ameliorated through professional support e.g., supervision [26]. A recent systematic review of 32 studies concluded effective clinical supervision was associated with lower burnout and greater staff retention [10]. The QuEDS F-PGS study did not measure impacts on burnout or staff retention, however, QuEDS FPGS participants reported feeling 'less stressed' and 'greater enjoyment' in their ED-specific clinical work. Inference of these reported outcomes in addition to reported 'increased involvement in service development activities' may translate to positive impacts on clinician burnout, staff retention and broader positive service outcomes which should be explored in future studies.

Peer group supervision (PGS) is highly familiar to the Queensland Health allied health workforce. QuEDS F-PGS participants reported a preference for QuEDS F-PGS versus PGS. QuEDS F-PGS rated second only to individual supervision (50\%) as a preferred mode of clinical support/upskilling. PGS was rated less favourably by QuEDS F-PGS participants, only ranking higher than the least preferred option of online education. This differs markedly from Denman et al [3] who reported preferred upskilling for dietitians working in EDs in the following order: online webinars, workshops, online courses, followed by clinical supervision (15\%), work shadowing and professional interest groups. This may illustrate the historically poor uptake of professional supervision in the dietetic workforce, with anecdotally, a higher acceptance of the role of clinical supervision for dietitians who are working within the ED arena. Intention to continue with group supervision was not measured/reported in the PGS-AH or GCS evaluations $[15,11]$. In the present study, QuEDS F-PGS survey respondents indicated they would recommend F-PGS to other dietitians and $96 \%$ planned to continue with the program, a positive indicator of program sustainability.

The strength of this study is its appraisal of the QuEDS F-PGS model across the four Kirkpatrick levels for training evaluation and an examination of program sustainability through the RE-AIM lens. Inclusion of a baseline survey in addition to the follow up survey enabled us to measure significant positive change in confidence to deliver 'evidence-based care' and to 'engage with clients' over the first six months of participation in QuEDS F-PGS. The study also had a high response rate to the voluntary, anonymous surveys ( $70 \%$ for the LCP survey) which decreased inherent self-selection bias. Results analyses were conducted by a third party (NM) who did not take any part in the QuEDS F-PGS program. A broader examination of implementation of QuEDS F-PGS via the RE-AIM framework provided positive data with respect to reach, efficacy and implementation, however, broader adoption of the program has not been tested, nor maintenance of impact/outcomes post-completion of the F-PGS groups. Maintenance post completion was deliberately not measured as it is considered that supervision is an ongoing process throughout a clinical career and, therefore, F-PGS groups are ongoing. The comparative studies have confined discussion to efficacy and acceptability of the group supervision programs. Success of uptake of the QuEDS F-PGS model by other groups should be evaluated in future. 
Limitations in this study include the use of self-assessment measures in the surveys rather than direct measurement of clinical practice changes, impacts on patient or service outcomes. Other studies that evaluated group supervision programs $[11,15,23,24]$ have similarly utilised self-assessment questionnaires - the Clinical Supervision Evaluation Questionnaire (CSEQ) [20] - a validated tool, with additional semi-structured interviews [23], focus groups [11], or free text comments [24] for additional data collection. This study used surveys which were not validated but were informed by CSEQ and the Kirkpatrick 4 Level Training Evaluation framework with additional free text comments. This was a considered decision by the authors to fully consider data collection that could influence future refinements to the QuEDS F-PGS model.

\section{Conclusion}

The importance of this study is the unique insight it provides into provision of group supervision for dietitians working in EDs. QuEDS F-PGS is a cost-effective, accessible, online model of peer group support that enables dietitians working within EDs to improve clinical skills, confidence and take a more active role in advocacy for optimal ED patient care and in ED-specific service development.

The author hypothesises the overall success of QuEDS F-PGS is due to its high level of structure, fidelity to the model assured by the Lead Facilitator and Facilitator roles, ongoing participant feedback and implementation of adult learning theory principles to create a safe, learning environment.

Evaluation of the implementation and impact of QuEDS F-PGS model of peer group support has shown that a cost-effective framework of peer group support can overcome the usual challenges to implementation of supervision, positively impact the dietetic ED workforce and organisations, and by inference the dietetic care of people with eating disorders. Here, we provided a detailed description of a peer supervision model with high acceptability and reported effectiveness which could be adapted for use in other specialist areas and other health professional groups. Consideration should be given to broader testing of QuEDS F-PGS model for other health professional groups working in eating disorders and other specialist clinical areas within dietetics. Consideration should also be given to direct measurement of patient and service outcomes and how to ameliorate facilitator-based outcome variables.

In view of the highly positive evaluation of the QuEDS F-PGS model and its outcomes, which align with the ANZAED clinical practice standards stated aims for clinical supervision, and its cost-effectiveness, it is possible to conclude that QuEDS F-PGS model of peer group support is an appropriate adjunct, or alternative, for individual clinical supervision.

'QuEDS F-PGS has been a wonderful support to my practice, I have learnt new skills and had exposure to wide variety of focus topics and PD opportunities. It has also been great in connecting with other ED dietitians and reduced feelings of isolation within the field. I would highly recommend it to anyone seeking eating disorders supervision.'

Private dietitian participant in F-PGS D 


\section{Abbreviations}

ANZAED: Australia and New Zealand Academy of Eating Disorders; ED: Eating Disorder; QuEDS: Queensland Eating Disorder Service; F-PGS: Facilitated Peer Group Support; CSEQ: Clinical Supervision Evaluation Questionnaire; PGS: Peer Group Supervision; EOI: Expression of Interest; DNSC: Dietitian Nutritionist Strategic Coalition; COD-ED: Collaboration of Dietitians in Eating Disorders; QHNDLC: Queensland Health Nutrition and Dietitian Leadership Coalition; LCP: Learning and Clinical Practice Survey; RE-AIM: Reach, Efficacy, Adoption, Implementation, Maintenance; HHS: Hospital and Health Service; GCS: Group Clinical Supervision; PGS-AH: Peer Group Supervision for Allied Health; PGS-RT: Peer Group Supervision for Radiation Therapists

\section{Declarations}

\section{Acknowledgements}

The authors would like to thank Amy Davis, Emma Coleman, Lucinda Morrow, Claire Gardiner, Emily Twine and Shane Jeffrey who were involved in facilitation roles, and Dr Kylie Burke for her support.

\section{Authors' contributions}

AD devised and developed the F-PGS model, wrote the program documentation suite, designed the program evaluation, and wrote the first draft of the manuscript. NM assisted with the development of the LCP survey, analysed the data, and contributed to writing the manuscript. WW and AH provided departmental support for the program and critically reviewed the manuscript. ET critically reviewed the manuscript.

\section{Funding}

No sources of funding to declare.

\section{Availability of data and materials}

The datasets generated and analysed during this study are available from the corresponding author on reasonable request.

\section{Ethics approval and consent to participate}

Ethics exemption for evaluation of the broader roll out of the QuEDS model of F-PGS was sought and granted by the Royal Brisbane and Women's Hospital Human Research Ethics Committee. Ref no: LNR/2019/QRBW/59576. The project was deemed compliant with 'Ethical Considerations in Quality Assurance and Evaluation Activities' 2014.

\section{Consent for publication}


Not applicable.

\section{Competing interests}

All authors declare that they have no competing interests.

\section{References}

1. The Butterfly Foundation. National agenda for eating disorders 2017 to 2022: Establishing a baseline of evidence-based care for any Australian with or at risk of an eating disorder. Sydney: The Butterfly Foundation; 2018. Available from https://www.butterfly.org.au/wpcontent/uploads/2020/05/National-Agenda-for-Eating-Disorders-2018.pdf.

2. Heruc G, Hurst K, Casey A, Fleming K, Freeman J, Fursland A, et al. ANZAED eating disorder treatment principles and general clinical practice and training standards. J Eat Disord. 2020;8:1-9.

3. Denman E, Parker EK, Ashley MA, Harris DM, Halaki M, Flood V, et al. Understanding training needs in eating disorders of graduating and new graduate dietitians in Australia: an online survey. $\mathrm{J}$ Eat Disord. 2021;9:27.

4. Dawson M, Phillips B, Leggat SG. Clinical supervision for allied health professionals: J Allied Health. 2013;42(2):65-73.

5. Lakeman R, McIntosh C. Perceived confidence, competence and training in evidence-based treatments for eating disorders: a survey of clinicians in an Australian regional health service. Australas Psychiatry. 2018:26(4);432-436.

6. Maguire S, Li A, Cunich M, Maloney D. Evaluating the effectiveness of an evidence-based online training program for health professionals in eating disorders. J Eat Disord. 2019;7(1).

7. Heruc G, Hart S, Stiles G, Fleming K, Casey A, Sutherland F, et al. ANZAED practice and training standards for dietitians providing eating disorder treatment. J Eat Disord. 2020;8:77.

8. Pellizzer ML, Waller G, Wade TD. A pragmatic effectiveness study of 10-session cognitive behavioural therapy (CBT-T) for eating disorders: targeting barriers to treatment provision. Eur Eat Disord Rev. 2019;27:557-70.

9. Heafala A, Ball L, Rayner J, Mitchell LJ. What role do dietitians have in providing nutrition care for eating disorder treatment? An integrative review. J Hum Nutr Diet. 2021;00:1-12.

10. Martin P, Lizarondo L, Kumar S, Snowdon D. Impact of clinical supervision on healthcare organisational outcomes: A mixed methods systematic review. PLoS ONE. 2021;16(11): e0260156.

11. Gardner M, McKinstry C, Perrin B. Group clinical supervision for allied health professionals. Aust J Rural Health. 2021;29:538-548.

12. Kirk SF, Eaton J, Auty L. Dietitians and supervision: should we be doing more? J Hum Nutr Diet. 2000;13(5):317-22.

13. Davis AM, Davis A. Solving the supervision dilemma for dietitians working in eating disorders innovative programmes from a statewide advisory service. J Eat Disord. 2019;7(Suppl 1):07. 
14. Brownlow RS, Maguire S, O’Dell A, Dias-da-Costa C, Touyz S, Russell J. Evaluation on an online training program in eating disorders for health professionals in Australia. J Eat Disord. 2015;3:37.

15. Kuipers P, Pager S, Bell K, Hall F, Kendall M. Do structured arrangements for multidisciplinary peer group supervision make a difference for allied health professional outcomes? J Multidiscip Healthc. 2013;6:391-397.

16. Terry D, Nguyen H, Perkins AJ, Peck B. Supervision in healthcare: a critical review of the role, function, and capacity for training. Univers J Public Health. 2020;8(1):1-14.

17. Knowles SM. Innovations in teaching styles and approaches based on adult learning. J Ed Soc Work. 2013;8(2):1972.

18. New Zealand Coaching \& Mentoring Centre. The power of peer supervision. Tools for supervision \& mentoring groups. 2012. Available from https://www.coachingmentoring.co.nz

19. Gaglio B, Shoup JA, Glasgow RE. The RE-AIM Framework: A systematic review of use over time. Am J Public Health. 2013;103(6):e38-e46.

20. Horton S, de Lourdes Drachler M, Fuller A, de Carvalho Leite JC. Development and preliminary validation of a measure for assessing staff perspectives on the quality of clinical group supervision. Int J Lang Commun Disord. 2008;43(2):126-134.

21. Kirkpatrick, J. D., Kirkpatrick, W. K. Kirkpatrick's Four Levels of Training Evaluation. United States: ATD Press;2016.

22. Dawber C, O'Brien T. A longitudinal, comparative evaluation of reflective practice groups for nurses working in intensive care and oncology. J Nurs \& Care. 2013;3:138.

23. Pager SC, Kuipers P, Bell K, Hall F. Evaluation of a peer group model of supervision for the allied health workforce in Queensland: a descriptive overview. Internet J Allied Health Sci Prac. 2018;Apr 05,16(2).

24. Dungey G, Neser, H, Sim D. New Zealand radiation therapists' perceptions of peer group supervision as a tool to reduce burnout symptoms in the clinical setting. J Med Radiat Sci. 2020;67(3):225-232.

25. Reschke DJ, Dawber C, Millear PM, Medoro L. Group clinical supervision for nurses: process, group cohesion and facilitator effect. Aus J Adv Nurs. 2020;38(3):66-74.

26. Warren CS, Schafer KJ, Crowley ME, Olivardia R. A qualitative analysis of job burnout in eating disorder treatment providers. Eat Disord. 2012;20(3):175-195.

\section{Figures}




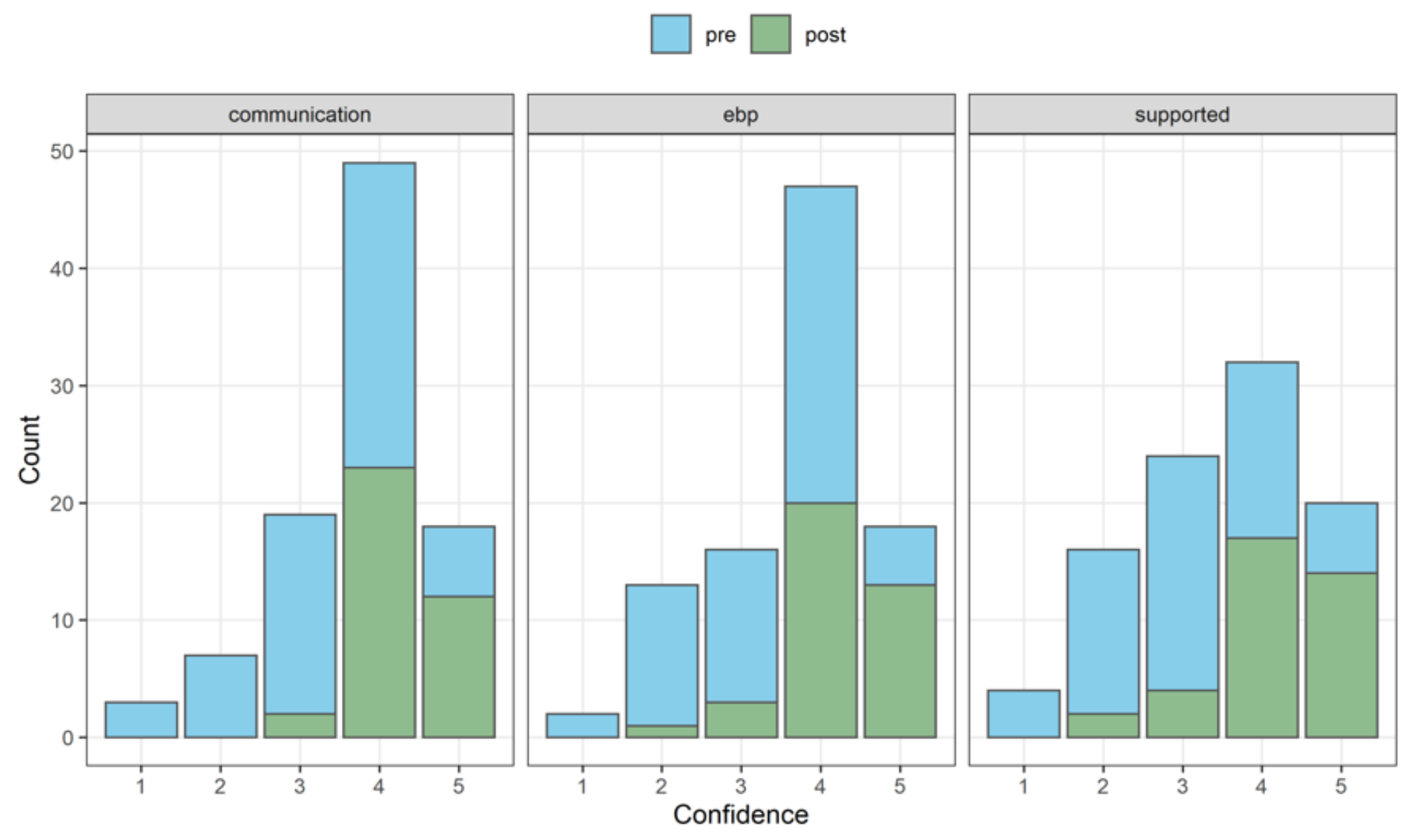

\section{Figure 1}

Pre-post changes in ratings (baseline to follow-up survey) of confidence to communicate with and provide evidence-based dietetic interventions (EBP) for people with eating disorders, and of feeling supported to work as a dietitian in the field of eating disorders.

\section{Supplementary Files}

This is a list of supplementary files associated with this preprint. Click to download.

- Additionalfile1sessionformatwithscript.pdf

- Additionalfile2facilitatorsguide.pdf

- Additionalfile3baselinesurvey.pdf

- Additionalfile4followupsurvey.pdf

- Additionalfile5LCPsurvey.pdf

- Additionalfile6surveydevelopment.pdf 